\title{
Telephone-based management of pressure ulcers in people with spinal cord injury in low- and middle-income countries: a randomised controlled trial
}

\author{
M Arora ${ }^{1,2}$, LA Harvey ${ }^{1,2}$, JV Glinsky ${ }^{1,2}$, HS Chhabra ${ }^{3}$, S Hossain ${ }^{4}$, N Arumugam ${ }^{5}$, PK Bedi ${ }^{5}$, L Lavrencic ${ }^{6}$, \\ AJ Hayes ${ }^{7}$ and ID Cameron ${ }^{1,2}$
}

Study design: A multicentre, prospective, assessor-blinded, parallel randomised controlled trial.

Objectives: The objective of the trial was to determine the effectiveness of telephone-based management of pressure ulcers in people with spinal cord injury (SCl) in low- and middle-income countries.

Methods: One hundred and twenty people with $\mathrm{SCl}$ living in the community were recruited through three hospitals in India and Bangladesh between November 2013 and March 2016. Participants had sustained an SCl >3 months prior and had a pressure ulcer. Participants were randomly allocated (1:1) to a control or intervention group. Participants in the control group received no intervention. Participants in the intervention group received weekly advice by telephone for 12 weeks about the management of their pressure ulcers from a trained health-care professional. Outcomes were measured by a blinded assessor at baseline and 12 weeks. There was one primary outcome, namely, the size of the pressure ulcer and 13 secondary outcomes.

Results: The mean between-group difference for the size of the pressure ulcer at 12 weeks was $2.3 \mathrm{~cm}^{2}$ (95\% confidence interval -0.3 to 4.9 ; favouring the intervention group). Eight of the 13 secondary outcomes were statistically significant.

Conclusion: The results of our primary outcome (that is, size of pressure ulcer) do not provide conclusive evidence that people with $\mathrm{SCl}$ can be supported at home to manage their pressure ulcers through regular telephone-based advice. However, the results from the secondary outcomes are sufficiently positive to provide hope that this simple intervention may provide some relief from this insidious problem in the future.

Spinal Cord (2017) 55, 141-147; doi:10.1038/sc.2016.163; published online 20 December 2016

\section{INTRODUCTION}

Pressure ulcers following spinal cord injury (SCI) are common with serious consequences. ${ }^{1,2}$ They can cause sepsis, osteomyelitis, amputation and permanent scarring. ${ }^{3}$ They are also associated with depression, pain and social isolation, which negatively impact quality of life. ${ }^{4}$ They prevent people from working and therefore have large financial implications for individuals and their families. Pressure ulcers are also a major cause of mortality, particularly in low- and middle-income countries (LMICs) where there is limited access to treatment options. ${ }^{5}$ A large cohort study from Bangladesh reported that one in five people with SCI who were wheelchair dependent had died within 2 years of discharge from hospital, mostly from pressure ulcers (2-year survival was $81 \%$; $95 \%$ confidence interval (CI) $76-86 \%) .{ }^{6}$ Given the high rates of postdischarge mortality in LMICs, finding effective, low-cost and locally applicable treatment options for the management of pressure ulcers is a key part of improving levels of care for people with SCI globally. ${ }^{5}$

There is a large divide between the management of pressure ulcers in high-income countries compared with the management in LMICs. ${ }^{5}$
Treatment of pressure ulcers in high-income countries is intensive and multi-faceted. It involves hospitalisation or home nursing and regular wound care as well as specialised equipment, such as alternating pressure-relieving mattresses. ${ }^{3,7-9}$ However, such resource-intensive treatments are not common options in LMICs, ${ }^{10}$ where economic constraints mean that people with SCI are largely left to manage their pressure ulcers alone at home. ${ }^{11}$ Regular professional advice over the telephone might be a realistic and low-cost option, which may make a notable difference to those living in LMICs with no or limited access to health care. The accessibility of mobile phones in most LMICs now enables cheap and simple communication between a health-care professional and those living with an SCI. ${ }^{12}$ A small number of studies have looked at telephone-based management of pressure ulcers. ${ }^{13,14}$ However, these studies were conducted in high-income countries where the telephone-based management was an adjunct to an already high level of care.

We hypothesised that a health-care professional could help people with pressure ulcers to manage their pressure ulcers at home through weekly telephone contact. Our hope for this intervention was based on

\footnotetext{
${ }^{1}$ John Walsh Centre for Rehabilitation Research, Kolling Institute of Medical Research, Northern Sydney Local Health District, St Leonards, New South Wales, Australia; ${ }^{2}$ Sydney Medical School Northern, University of Sydney, Sydney, New South Wales, Australia; ${ }^{3}$ Department of Spine Service, Indian Spinal Injuries Centre, Delhi, India; ${ }^{4}$ Centre for Rehabilitation of the Paralysed, Savar, Bangladesh; ${ }^{5}$ Department of Physiotherapy, Punjabi University, Patiala, India; ${ }^{6}$ Spinal Cord Injury Unit, Royal North Shore Hospital, St Leonards, New South Wales, Australia and ${ }^{7}$ School of Public Health, University of Sydney, Sydney, New South Wales, Australia

Correspondence: Professor LA Harvey, John Walsh Centre for Rehabilitation Research, Kolling Institute of Medical Research, Level 13, Sydney Medical School Northern, The University of Sydney, Reserve Road, St Leonards, New South Wales 2065, Australia.

E-mail: lisa.harvey@sydney.edu.au

Received 25 July 2016; revised 28 September 2016; accepted 6 October 2016; published online 20 December 2016
} 
two trials indicating the benefits of patient education and support for the management of pressure ulcers. ${ }^{15,16}$ Typically, education and support are provided through face-to-face contact with a health-care professional, but this is a costly service and hence not feasible for most in LMICs. In LMICs, high-quality education and support could be provided over the telephone. If effective, this would provide some relief from the insidious problem of pressure ulcers in LMICs at little cost. The aim of this trial was to determine the effects of a 12-week telephone-based pressure ulcer management programme on pressure ulcer size in people with SCI. The secondary aims were to determine the effects of telephone-based management on outcomes reflecting healing of pressure ulcers, participants' and clinicians' perspective of treatment effectiveness, quality of life and depression.

\section{METHODS}

\section{Design}

A multicentre, prospective, assessor-blinded, parallel, randomised controlled trial was undertaken in India and Bangladesh. People living in the community with pressure ulcers secondary to SCI were randomised to a control or intervention group. Participants in both groups managed their pressure ulcers alone at home. However, participants in the intervention group also received weekly advice over the telephone for 12 weeks about the management of their pressure ulcers from a trained health-care professional. Participants were recruited between November 2013 and March 2016. The last 12-week assessment took place in May 2016. The trial protocol (including full details of the trial rationale, design and statistical analysis) has been published and is available online. ${ }^{17}$

The trial was conducted through the Indian Spinal Injuries Centre (New Delhi, India), Punjabi University (Punjab, India) and The Centre for Rehabilitation of the Paralysed (Dhaka, Bangladesh). Initially, the trial was planned for just one site. However, the two other sites were entered into the trial 8 months later to increase the rate of recruitment. The trial was approved by the Institutional Ethics Committees at each site. We certify that all applicable institutional and governmental regulations concerning the ethical use of human volunteers were followed during the course of this research. The trial was prospectively registered at the Australian New Zealand Clinical Trial Registry (www.anzctr.org.au; Identifier: ACTRN12613001225707).

\section{Participants}

Participants were recruited from outpatient clinics and through hospital databases. Participants were included if they:

- were aged > $>18$ years;

- had sustained an SCI >3 months prior to recruitment;

- had at least one pressure ulcer on the sacrum, ischial tuberosity or greater trochanter of the femur;

- were unlikely to be admitted to hospital for management within the next 12 weeks;

- were living in the community;

- were able to speak either sufficient Hindi (for the Indian sites) or Bengali (for the Bangladesh site) to allow them to participate in the trial without the assistance of a translator;

- had access to a phone;

- had the potential to benefit from advice provided over the telephone.

Participants were excluded if they:

- had cognitive or verbal impairments;

- had any clinically significant medical condition that would compromise participation in the trial;

- were unable to be assessed at 12 weeks.

A computer-generated random allocation schedule was formulated prior to commencement of the trial by an independent person located in Australia who was not involved in recruitment. The rand() function in Excel was used to generate the schedule. ${ }^{18}$ A blocked allocation (1:1) schedule was used. Participants' allocations were placed in opaque, sequentially numbered and sealed envelopes that were kept in Australia. After a participant passed the screening process and completed the baseline assessment, a randomisation envelope was opened and allocation revealed. The participant was considered to have entered the trial at this point.

\section{Intervention}

Participants from both the control and intervention groups managed their pressure ulcers alone at home but typically with the assistance of family members. They were all given a pamphlet containing information about pressure ulcer management at the time of recruitment, and were free to seek any help or medical assistance that they deemed appropriate or could access. In addition, participants allocated to the intervention group received weekly phone calls from a trained health-care professional for 12 weeks. Each time the healthcare professionals rang the participants, they reinforced self-help strategies important for managing pressure ulcers, minimising psychological stress and enhancing engagement with life. Specifically, participants and their families received education and advice about appropriate seating, bed overlays, cushions, equipment, diet, nutrition and wound dressings. In addition, they were advised about techniques to relieve pressure and when to seek further medical or nursing attention. Some participants were advised to remain on strict bed rest. Participants were also provided with advice to help minimise dampness associated with any bladder or bowel incontinence. Each week, participants and the health-care professionals negotiated goals that were reviewed at the next phone call. For example, a participant's goal might have included strict bed rest for the next week.

Two health-care professionals provided the advice over the telephone. One was a nurse and the other was a physiotherapist. They both had extensive experience in the management of SCI and pressure ulcers in LMICs. In addition, they were supported by multidisciplinary teams specialised in SCI and pressure ulcers from Australia, India and Bangladesh.

\section{Outcome measures}

All assessments were conducted at the beginning and end of the 12-week trial period by three independent, trained, blinded assessors (one at each site). The assessments were either conducted at the hospital or in participants' homes. Participants were asked not to discuss their group allocation with assessors. The success of blinding was verified at the end of each participant's assessment by asking assessors to reveal whether they had been unblinded to group allocation.

The primary outcome was the size of the pressure ulcer at 12 weeks. This was assessed using commercially available grid paper designed for measuring pressure ulcers. Length and width were measured and expressed as $\mathrm{cm}^{2}$. If a participant had more than one pressure ulcer, the pressure ulcer with most potential to benefit by telephone-based advice was identified at baseline. This pressure ulcer was measured at baseline and 12 weeks.

There were 13 secondary outcomes. ${ }^{19-28}$ The details for each outcome are described in the freely available trial protocol ${ }^{17}$ and summarised in Table 1. One of the 13 secondary outcomes was time to healing. This outcome was collected from an unblinded assessor who rang all participants every fortnight and asked them if their pressure ulcer had healed. At this time, participants were also asked about costs associated with managing their pressure ulcers (details will be reported in a subsequent paper devoted to an economic analysis).

\section{Sample size}

There were very little data upon which to base power calculations. A sample of 120 people was therefore selected primarily on the basis of available resources. The minimally worthwhile treatment effect was set a priori as equivalent to $10 \%$ of the mean initial size of participants' pressure ulcers at baseline expressed in raw units $\left(\mathrm{cm}^{2}\right)$.

\section{Data analysis}

All data were entered twice onto an electronic database using the Research Electronic Data Capture (REDCap) software hosted at the University of Sydney, 
Table 1 Details of secondary outcomes

Outcome

PUSH

Depth of PU

Undermining distance of PU

Braden scale

HADS

Participation items-WHODAS

Utility score-EQ-5D-5L

Self-rated health EQ-5D-VAS

Participants' impression of PU status

Participants' confidence to manage PU

Clinician's impression of PU status

Participants' satisfaction

Self-report time for PU resolution
Description

The PUSH version 3.0 was used to measure the severity of the PU. ${ }^{19}$ The PUSH rates pressure ulcers on a scale of $0-17$ points taking into account the area of the PU, amount of exudate and type of tissue damage (lower value indicates less severity). Depth of the PU was measured using a sterile scaled probe at the centre of the PU. ${ }^{20}$ This was recorded in centimetres (lower value indicates less depth).

Undermining of the PU was measured using a sterile scaled probe at 12 O'clock, 3 O'clock, 6 O'clock and 9 O'clock directions and then summed to derive one single score. ${ }^{21}$ This was recorded in centimetres (lower value indicates less undermining).

The Braden scale was used to measure the risk of developing a PU on a scale of 0-23 points using six domains ${ }^{22}$ (higher value indicates lower risk).

HADS was used to measure depression. ${ }^{23,24}$ HADS assesses depression on a scale of 0-21. A score of 0-7 indicates normal, 8-10 indicates borderline-abnormal and a score of 11-12 indicates abnormal (lower value indicates fewer depressive symptoms). Participation items on WHODAS 2.0 questionnaire were used to measure participation. ${ }^{25}$ The WHODAS rates participation over the past 30 days using an eight-item questionnaire. Each item is scored on a five-point scale ranging from none ( 1 point) to extreme/cannot do (5 points). The total score ranges from 8 to 40 points (lower value indicates higher participation).

Participants' health state was measured using the five dimensions on the EQ-5D-5L health survey. ${ }^{26,27}$ The five dimensions are mobility, self-care, usual activities, pain/depression and anxiety/depression. These were used to derive a utility score (higher value indicates higher health state).

Self-rated health was measured using a health thermometer on the EQ-5D-5L health survey. ${ }^{26,27}$ Participants were asked to rate their health on a vertical VAS numbered from 0 (worst imaginable health state) to 100 (best imaginable health state; higher value indicates higher health).

Participants' impression of PU status was assessed with one question, namely- 'How would you rate the quality of your wound and skin over the affected area?' Participants were asked to respond to this question using a 10-point NRS ${ }^{28}$ anchored at one end with 'extremely poor' and at the other end with 'extremely good' (higher value indicates a good impression of PU status).

Participants' confidence to manage their PUs was assessed with one question, namely_-How confident are you in managing your PU at home each day?' Participants were asked to respond to this question using a 10-point NRS ${ }^{28}$ anchored at one end with 'not very confident' and at the other end with 'very confident' (higher value indicates more confidence).

Clinicians' impression of PU status was assessed with one question, namely - 'How would you rate the quality of the wound and skin over the affected area?' The blinded assessor was asked to respond to this question using a 10-point NRS ${ }^{28}$ anchored at one end with 'extremely poor' and at the other end with 'extremely good' (higher value indicates a good impression of PU status). Participants' satisfaction for health-care provision was assessed with one question, namely - 'How satisfied are you with the services you have received for your pressure ulcer over the past 12 weeks?' Participants were asked to respond to this question using a 10-point $\mathrm{NRS}^{28}$ anchored at one end with 'very unsatisfied' and at the other end with 'very satisfied' (higher value indicates more satisfaction with the services).

Self-report time for PU resolution was assessed on a fortnightly basis through one question, namely-'Is your pressure ulcer healed?' These data were collected fortnightly over the telephone and reported as 'yes' or 'no'. The total number of days to PU healing were calculated (fewer days indicates shorter time to healing).

Abbreviations: EQ-5D-5L, Euro Quality of Life 5-dimensional 5-level; HADS, Hospital Anxiety and Depression Scale; NRS, Numeric Rating Scale; PU, pressure ulcer; PUSH, Pressure Ulcer Scale for Healing; VAS, Visual Analogue Scale; WHODAS, World Health Organisation Disability Assessment Schedule.

Note: All the self-report questionnaires were administered to participants in English, Hindi or Bengali.

Australia. ${ }^{29}$ REDCap is a secure web application designed to support data capture for research studies. The data were analysed using intention to treat and the Stata software (Version 13, StataCorp LP, College Station, TX, USA). The analyses were conducted by a blinded researcher based at the John Walsh Centre for Rehabilitation Research, University of Sydney, Australia. Linear regression was used to determine the mean between-group differences (and 95\% CIs) for all continuous outcomes using baseline scores and group allocation as covariates. Missing data were not imputed. In addition, time to pressure ulcer healing was analysed using survival analysis and Cox's regression, with the treatment effect expressed as an unadjusted Hazard ratio (and 95\% CI). $P$-values $<0.05$ were considered significant.

\section{RESULTS}

\section{Flow of participants}

A total of one hundred and twenty people with pressure ulcers secondary to SCI were recruited through the Indian Spinal injuries Centre $(n=69)$, the Centre for Rehabilitation of the Paralysed $(n=40)$ and an outpatient clinic at the Punjabi University $(n=11)$. The flow of participants through the trial is shown in Figure 1. Five participants dropped out of the trial prior to the 12-week assessment.

\section{Participant characteristics}

The mean (s.d.) age and time since injury were 35 years (12) and 7 years (13), respectively. Participants had a mix of complete and incomplete SCI according to the International Standards for the Neurological Classification of SCI. ${ }^{30}$ Participants had stage $2(n=35)$, stage $3(n=83)$ and stage $4(n=2)$ pressure ulcers. ${ }^{7}$ These pressure ulcers were located on the sacrum $(n=54)$, ischial tuberosity $(n=45)$ and greater trochanter of the femur $(n=21)$. The groups were similar at baseline (see Table 2).

\section{Compliance with trial protocol}

There was one protocol violation with the inclusion of a participant who had sustained his SCI $<3$ months prior to randomisation. The data for this participant were included in all analyses. Adherence to all other aspects of the trial protocol was very good. The protocol dictated that all participants be assessed in person at baseline and at 12 weeks; however, two participants were assessed only by telephone at 12 weeks. For one participant, the pressure ulcer had been surgically repaired and was deemed healed at 12 weeks, and in the other participant, the blinded assessor instructed a family member over the telephone to 


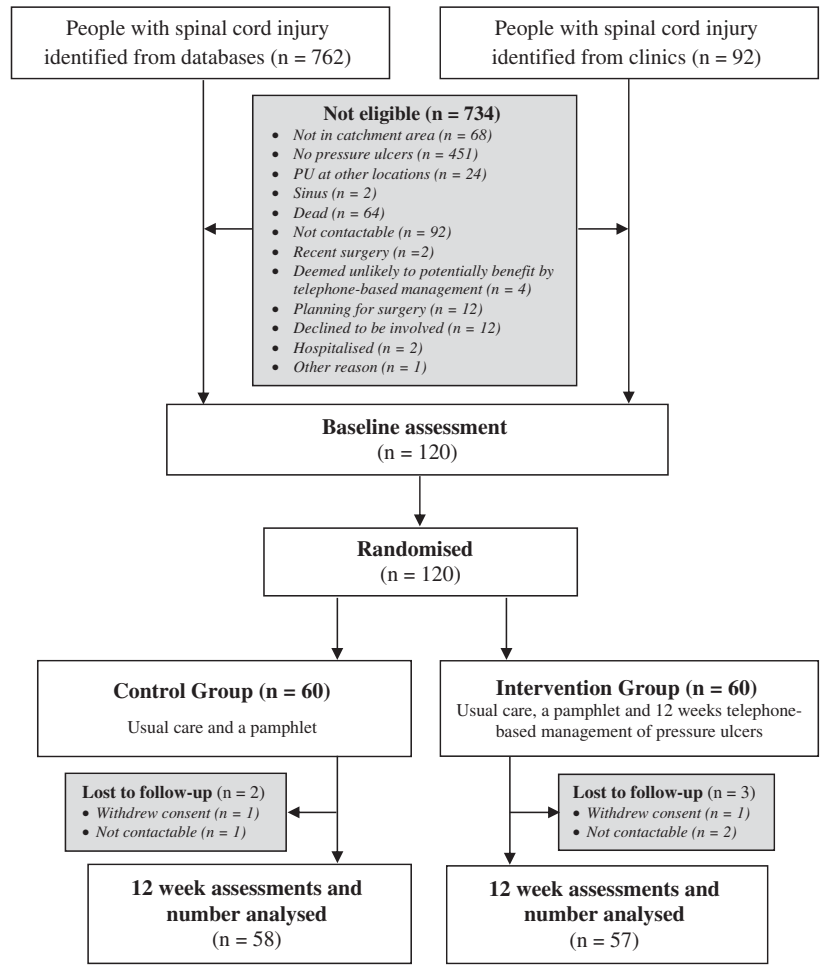

Figure 1 Design and flow of participants through the trial.

take the various pressure ulcer measurements. All other outcomes for these two participants were collected by interview over the telephone. There was 100\% success at keeping assessors blinded.

A mean (s.d.) of 11 (2) phone calls were made to each participant in the intervention group. That is, a total of 685 of the planned 720 telephone calls (this does not include the multiple attempts often required to make contact with a participant). The mean (s.d.) duration of the phone calls was 16 (2) min.

\section{Treatment effect}

Primary outcome: The mean between-group difference for the size of the pressure ulcer at 12 weeks, adjusted by baseline size, was $2.3 \mathrm{~cm}^{2}$ favouring the intervention group ( $95 \% \mathrm{CI}-0.3$ to 4.9 ; $P=0.08$; see Table 3). The minimally worthwhile treatment effect (equivalent to $10 \%$ of mean initial values) was $1.1 \mathrm{~cm}^{2}$.

Secondary outcomes: The mean between-group differences (95\% CI and $P$-values) for the secondary outcomes are presented in Table 3. The Kaplan-Meier estimates for time to healing of the pressure ulcers for both groups and accompanying hazard ratio (95\% CI) are depicted in Figure 2.

\section{DISCUSSION}

This trial is the first randomised controlled trial to investigate a low-cost treatment option for the management of pressure ulcers in people with SCI living in LMICs. Pressure ulcers for this group of people are a major cause of mortality and misery and receive very little research attention. ${ }^{6,31}$ The primary aim of this trial was to determine the effects of a 12-week telephone-based pressure ulcer management programme on pressure ulcer size in people with SCI. The results for the primary outcome (size of pressure ulcer) failed to provide a clear answer to our research question; however, the results do not rule out the possibility that advice about pressure ulcer management provided
Table 2 Baseline characteristics of participants

\begin{tabular}{|c|c|c|}
\hline \multirow{2}{*}{ Participants' characteristics } & \multicolumn{2}{|c|}{ Randomised $(\mathrm{n}=120)$} \\
\hline & $\operatorname{Con}(n=60)$ & Int $(\mathrm{n}=60)$ \\
\hline Age (years), mean (s.d.) & $36(12)$ & $35(11)$ \\
\hline \multicolumn{3}{|l|}{ Gender, $n(\%)$} \\
\hline Male & $54(90)$ & $52(87)$ \\
\hline Female & $6(10)$ & $8(13)$ \\
\hline Duration since injury (years), median (IQR) & $3.7(1.3,8.5)$ & $3.7(1.4,9.6)$ \\
\hline \multicolumn{3}{|l|}{ Mode of injury, $n(\%)$} \\
\hline Road traffic accident & $23(38)$ & $32(53)$ \\
\hline Fall from height & $19(32)$ & $18(30)$ \\
\hline Others & $15(25)$ & $10(17)$ \\
\hline Unknown & $3(5)$ & - \\
\hline \multicolumn{3}{|l|}{ Neurological level of injury, $n(\%)$} \\
\hline Upper cervical (C1-C4) & $4(7)$ & $1(2)$ \\
\hline Lower cervical (C5-C8) & $13(22)$ & $11(19)$ \\
\hline Upper thoracic (T1-T9) & $14(23)$ & $17(28)$ \\
\hline Lower thoracic (T10-L1) & $23(38)$ & $21(35)$ \\
\hline Lumbar and sacral & - & $2(3)$ \\
\hline Unknown & $6(10)$ & $8(13)$ \\
\hline \multicolumn{3}{|l|}{ ASIA impairment scale, $n(\%)$} \\
\hline$A$ & $47(78)$ & $52(87)$ \\
\hline $\mathrm{B}$ & $2(3)$ & $3(5)$ \\
\hline $\mathrm{C}$ & $8(14)$ & $2(3)$ \\
\hline D & $1(2)$ & - \\
\hline Unknown & $2(3)$ & $3(5)$ \\
\hline \multicolumn{3}{|l|}{ Location of PU, $n(\%)$} \\
\hline Sacral & $25(42)$ & $29(48)$ \\
\hline Right ischial tuberosity & $10(17)$ & $13(22)$ \\
\hline Left ischial tuberosity & $12(20)$ & $10(17)$ \\
\hline Right trochanter of the femur & $8(13)$ & $6(10)$ \\
\hline Left trochanter of the femur & $5(8)$ & $2(3)$ \\
\hline \multicolumn{3}{|l|}{ Stage of pressure ulcers, $n(\%)$} \\
\hline Stage II & $13(22)$ & $22(37)$ \\
\hline Stage III & $45(75)$ & $38(63)$ \\
\hline Stage IV & $2(3)$ & - \\
\hline
\end{tabular}

Abbreviations: ASIA, American Spinal Cord Injury Association; Con, control group Int, intervention group; IQR, interquartile range; PU, pressure ulcer.

over the telephone may be a worthwhile intervention (mean betweengroup difference $=2.3 \mathrm{~cm}^{2} ; 95 \% \mathrm{CI}-0.3$ to 4.9 ). The upper bound of the $95 \% \mathrm{CI}\left(4.9 \mathrm{~cm}^{2}\right)$ associated with the mean between-group difference is greater than our predetermined minimally worthwhile treatment effect of $1.1 \mathrm{~cm}^{2}$ (equivalent to $10 \%$ of mean initial values). This indicates that it would be valuable to repeat this trial with a larger sample particularly given that 8 of our 13 secondary outcomes indicate a statistically significant between-group difference.

The results of our survival analysis for time to healing are particularly promising, indicating a between-group difference favouring the intervention group (hazard ratio $=2.0 ; 95 \%$ CI $1.0-3.9$ ). However, these results need to be verified, because these data were collected from an unblinded assessor who rang all participants each fortnight and asked them if their pressure ulcers had healed. Such 
Table 3 Results of the intention-to-treat analysis for the primary and secondary outcomes at 12 weeks after randomisation

Baseline

$\operatorname{Con}(\mathrm{n}=58)$

Size of PU $\left(\mathrm{cm}^{2}\right)$

PUSH score/17 points

Depth of PU (cm)

Undermining distance of PU (cm)

Braden score (23 points)

Depression items-HADS (21 points)

Participation items-WHODAS (40 points)

Utility score-EQ-5D-5L (units)

Health rating-EQ-5D-VAS (100 points)

Participants' impression of

PU status (10 points)

Participants' confidence to

manage PU (10 points)

Clinician's impression of PU status (10 points)

Participants' satisfaction (10 points)

$\begin{array}{ccccc}12.5(13.2) & 9.2(11.6) & 8.1(10.5) & 4.2(7.1) & 2.3(-0.3 \text { to } 4.9 ; P=0.08) \\ 10.8(2.9) & 9.8(2.7) & 8.0(5.3) & 5.1(5.0) & 1.8(0.3 \text { to } 3.3 ; P=0.02) \\ 1.3(1.3) & 1.0(1.1) & 1.0(1.4) & 0.5(1.0) & 0.2(-0.1 \text { to } 0.5 ; P=0.17) \\ 2.1(4.4) & 2.0(4.1) & 2.2(4.5) & 1.5(3.9) & 0.6(-0.2 \text { to } 1.4 ; P=0.14) \\ 11.7(2.9) & 11.9(2.7) & 12.1(2.7) & 13.6(2.9) & 1.4(0.7 \text { to } 2.0 ; P<0.001) \\ 11.6(4.1) & 12.1(4.2) & 11.3(4.2) & 11.5(4.4) & 0.2(-1.1 \text { to } 1.4 ; P=0.77) \\ 27.5(5.5) & 26.4(5.7) & 27.3(5.4) & 24.4(4.9) & 2.3(0.8 \text { to } 3.8 ; P=0.003) \\ -0.47(0.29) & -0.52(0.21) & -0.39(0.38) & -0.37(0.36) & 0.1(0.02 \text { to } 0.2 ; P=0.01) \\ 52.5(18.4) & 52.8(15.4) & 62.8(19.2) & 73.1(16.4) & 10.5(4.5 \text { to } 16.6 ; P=0.001) \\ 4.5(1.9) & 4.4(1.6) & 6.2(2.6) & 6.9(2.6) & 0.8(-0.1 \text { to } 1.7 ; P=0.08) \\ 5.0(1.8) & & & & 1.7(1.0 \text { to } 2.3 ; P<0.001) \\ 4.5(1.3) & 5.2(2.0) & 5.6(2.1) & 7.3(1.6) & \end{array}$

(mean (95\% Cl; P-value)

Abbreviations: $\mathrm{Cl}$, confidence interval; Con, control group; EQ-5D-5L, Euro Quality of Life 5-dimensional 5-level; HADS, Hospital Anxiety and Depression Scale; Int, intervention group; PU, pressure ulcers; PUSH, Pressure Ulcer Scale for Healing; VAS, Visual Analogue Scale; WHODAS, World Health Organisation Disability Assessment Schedule.

Note: Primary outcome for the trial is in bold.

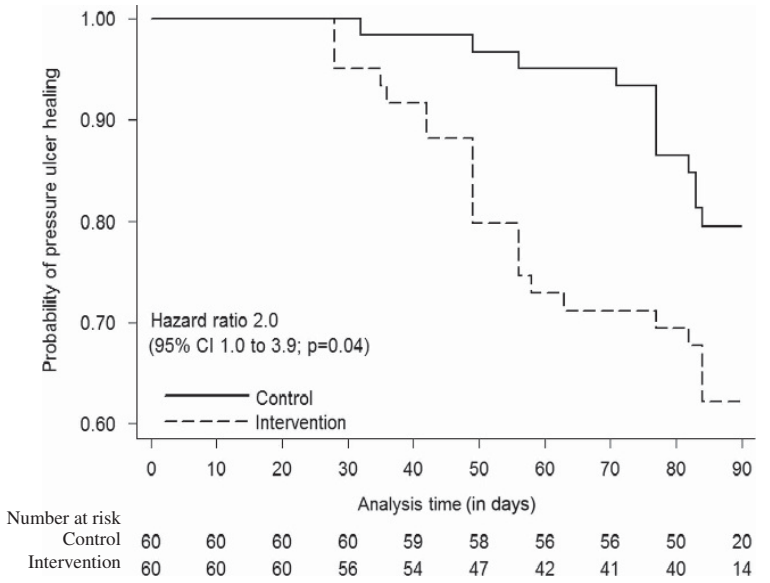

Figure 2 Kaplan-Meier survival estimates for pressure ulcer healing in the two groups.

self-report data may be biased because participants were unblinded and needed to use their own judgement as to whether their pressure ulcers had healed. Future studies could send blinded assessors to participants' homes each week, although this would be costly.

We demonstrated a statistically significant between-group difference for the Pressure Ulcer Scale for Healing (PUSH) although it is not clear whether the size of this treatment effect is clinically meaningful (mean between-group difference $=1.8$ points on a 17-point scale; $95 \%$ CI, $0.3-3.3$ points). We included the PUSH because it is one of the most widely used generic measures of pressure ulcer severity. ${ }^{7}$ It reflects the area of the pressure ulcer as well as amount of exudate and type of wound tissue. We did not use the PUSH as our primary outcome because we were concerned about its sensitivity with its limited ordinal scale. However, our reasonably precise estimate for the
PUSH indicates that these concerns were not justified. We also captured two other aspects of pressure ulcer severity, namely, pressure ulcer undermining and depth. The results for both these outcomes were inconclusive failing to rule in or out a potential therapeutic effect. Similarly, the participants' and clinicians' subjective impressions about whether the pressure ulcers had improved over the 12-week trial period were also inconclusive. Future studies could consider omitting some of these outcomes and instead relying on the PUSH, time to healing and size of pressure ulcer data to reflect the effectiveness of the intervention on pressure ulcers. Future studies could also consider following participants until the pressure ulcer is healed.

The results of the Braden scale are also interesting (mean betweengroup difference $=1.4$ points on a 23 -point scale, 95\% CI $0.7-2.0$ ). The Braden scale was included as an outcome measure to determine whether telephone-based pressure ulcer management changed the risk factors associated with developing a new pressure ulcer. The Braden scale rates six different indicators of susceptibility to pressure ulcers including sensation, moisture, activity, mobility, nutrition and friction or shear. The improvements on the Braden scale were primarily due to improved ratings on the moisture and nutrition indicators. This may be because the health-care professionals educated the participants about strategies to minimise moisture and enhance nutrition. The results of the Braden scale mirror participants' increased confidence in managing their pressure ulcers (mean between-group difference $=1.7$ points on a 10-point scale, 95\% CI 1.0-2.3). Participants in the intervention group rated their confidence with managing pressure ulcers highly (mean confidence $=7.3$ points on a 10 -point scale, s.d. 1.6). Together, these results may indicate that participants learnt about the importance of minimising moisture and maximising nutrition as well as other strategies for pressure ulcer management.

There is some indication that regular telephone-based management of pressure ulcers may have wider therapeutic benefits on both general health and participation, although it is not clear whether the size of the treatment effects are clinically meaningful. This is reflected in the 
statistically significant between-group differences for the World Health Organisation Disability Assessment Schedule (mean between-group difference $=2.3$ points on a 40 -point scale; $95 \%$ CI $0.8-3.8$ ), Euro Quality of Life-5 dimensional-5 level utility score (mean between-group difference $=0.1$ units; 95\% CI 0.02-0.2) and Euro Quality of Life-Visual Analogue Scale (mean between-group difference $=10.5$ points on a 100 -point scale; $95 \%$ CI 4.5-16.6), favouring the intervention. Participants may have reaped some social support from the regular contact with a concerned health-care professional. This may have been particularly valuable for the participants of this trial who were left to manage their pressure ulcers alone at home. The interpretation of these results is supported by participants' satisfaction with the telephone-based advice they received (mean level of satisfaction for the intervention group was 8.6 points on a 10-point scale, s.d. 1.4). Importantly, the participants in the intervention group were more satisfied than the participants in the control group (mean betweengroup difference 2.1 points on a 10 -point scale; 95\% CI 1.3-2.9) even though the participants in the control group were also somewhat satisfied (mean level of satisfaction for the control group was 6.4 points on a 10 -point scale; s.d. 2.7). The satisfaction of the control group may indicate that they valued the contact with the health-care professionals during the assessments.

We included a measure of depression to explore the possibility that regular telephone contact helps alleviate depression. However, there was no indication that this was the case (mean between-group difference $=0.2$ points on a 21 -point scale; $95 \%$ CI -1.1 to 1.4 ) even though mean scores on the Hospital Anxiety and Depression Scale at baseline are indicative of moderate depression. ${ }^{24}$ The failure of the intervention to reduce depression is not altogether surprising because the health-care professionals providing the telephone-based advice were not psychologists, and many participants were being encouraged to remain on bed rest. Prolonged bed rest probably does not help alleviate depression.

Intervention participants could not always follow the advice provided by the health-care professionals. For example, some participants were alone at home during the day without assistance and therefore could not regularly dress their pressure ulcers or change bed linen to remain dry. Others needed to get up and go to work because of the profound implications of limited income and social support for them and their families. Sometimes the problems were too great to solve by advice. For example, some participants needed surgery or wound debridement, and other participants did not have access to basic dressings, adequate nutrition, appropriate bed overlays or pressure cushions. A few participants had other associated conditions such as diabetes that were poorly managed and contributing to the pressure ulcers.

In all, telephone-based management of pressure ulcers is a promising intervention for LMICs. Although the results of our primary outcome (that is, size of pressure ulcer) do not provide conclusive evidence about the effectiveness of telephone-based management, they also do not rule out the possibility that this intervention may provide a feasible approach to this insidious problem. In addition, the results from the secondary outcomes are sufficiently positive to justify a larger randomised controlled trial with a longer intervention and follow-up.

\section{DATA ARCHIVING}

There were no data to deposit.

\section{CONFLICT OF INTEREST}

MA conducted this trial in partial fulfilment of his $\mathrm{PhD}$. He was supported through a Prime Minister's Australia Asia Post Graduate Research Endeavour Award for the duration of his $\mathrm{PhD}$ candidature. The trial was supported by the John Walsh Centre for Rehabilitation Research. The authors declare no conflict of interest in trial design, data collection and analysis, decision to publish, or preparation of the manuscript. Those supporting this research have no financial interests in the results. The authors and all organisations associated with the authors did not confer any benefit.

\section{ACKNOWLEDGEMENTS}

The authors acknowledge the assistance of the project coordinators (Mr Jaspreet Singh Sethi, Md Abdullah and Mr Ranjit Roy) at the trial sites, the participants and staff who assisted in recruiting participants.

1 Zakrasek EC, Creasey G, Crew JD. Pressure ulcers in people with spinal cord injury in developing nations. Spinal Cord 2015; 53: 7-13.

2 Kovindha A, Kammuang-Lue $P$, Prakongsai $P$, Wongphan T. Prevalence of pressure ulcers in Thai wheelchair users with chronic spinal cord injuries. Spinal Cord 2015; 53: 767-771.

3 Canadian best practice guidelines for the prevention and management of pressure ulcers in people with spinal cord injury. A resource handbook for clinicians. (Internet). 2013 (cited 05 June 2016). Available from http://onf.org/system/attachments/168/ original/Pressure_Ulcers_Best_Practice_Guideline_Final_web4.pdf.

4 Moore ZE, Cowman S. Repositioning for treating pressure ulcers. Cochrane Database Syst Rev 2015; 1: Cd006898.

5 Bickenbach J, Officer A, Shakespeare T, von Groote P (eds). World Health Organization and International Spinal Cord Society- International Perspectives on Spinal Cord Injury. WHO: Malta, 2013

6 Hossain MS, Rahman MA, Herbert RD, Quadir MM, Bowden JL, Harvey LA. Two-year survival following discharge from hospital after spinal cord injury in Bangladesh. Spinal Cord 2016; 54: 132-136.

7 European Pressure Ulcer Advisory Panel and National Pressure Ulcer Advisory Panel. Treatment of pressure ulcers: Quick Reference Guide. National Pressure Ulcer Advisory Panel: Washington, DC, USA, 2009.

8 Australian Wound Management Association. Pan Pacific Clinical Practice Guideline for the Prevention and Management of Pressure Injury. Cambridge Media: Osborne Park, WA, USA, 2012.

9 McInnes E, Jammali-Blasi A, Bell-Syer SEM, Dumville JC, Middleton V, Cullum N. Support surfaces for pressure ulcer prevention. Cochrane Database Syst Rev 2015; CD001735.

10 Dam A, Datta N, Mohanty UR, Bandhopadhyay C. Managing pressures ulcers in a resource constrained situation: a holistic approach. Indian J Palliat Care 2011; 17: 255-259.

11 Dieleman JL, Templin T, Sadat N, Reidy P, Chapin A, Foreman K et al. National spending on health by source for 184 countries between 2013 and 2040. Lancet 2016; 387: 2521-2535.

12 Swapna N (ed.). Telecommunication sector in India - an analysis. MPGI National Multi Conference; 7-8 April 2012; Maharashtra, India. International Journal of Computer Applications: Bangalore, India.

13 Halstead LS, Dang T, Elrod M, Convit RJ, Rosen MJ, Woods S. Teleassessment compared with live assessment of pressure ulcers in a wound clinic: a pilot study. Adv Skin Wound Care 2003; 16: 91-96.

14 Vesmarovich S, Walker T, Hauber RP, Temkin A, Burns R. Use of telerehabilitation to manage pressure ulcers in persons with spinal cord injuries. Adv Wound Care 1999; 12: 264-269.

15 Garber SL, Rintala DH, Holmes SA, Rodriguez GP, Friedman J. A structured educational model to improve pressure ulcer prevention knowledge in veterans with spinal cord dysfunction. J Rehabil Res Dev 2002; 39: 575-588.

16 Rintala DH, Garber SL, Friedman JD, Holmes SA. Preventing recurrent pressure ulcers in veterans with spinal cord injury: impact of a structured education and follow-up intervention. Arch Phys Med Rehabil 2008; 89: 1429-1441.

17 Arora M, Harvey LA, Hayes AJ, Chhabra HS, Glinsky JV, Cameron ID et al. Effectiveness and cost-effectiveness of telephone-based support versus usual care for treatment of pressure ulcers in people with spinal cord injury in low-income and middle-income countries: study protocol for a 12-week randomised controlled trial. BMJ Open 2015; 5: e008369.

$18 \mathrm{Kim}$ J, Shin W. How to do random allocation (randomization). Clin Orthop Surg 2014; 6: 103-109.

19 Hon J, Lagden K, McLaren AM, O'Sullivan D, Orr L, Houghton PE et al. A prospective, multicenter study to validate use of the PUSH in patients with diabetic, venous, and pressure ulcers. Ostomy Wound Manage 2010; 56: 26-36.

20 Van Lis MS, Van Asbeck FWA, Post MWM. Monitoring healing of pressure ulcers: a review of assessment instruments for use in the spinal cord unit. Spinal Cord 2010; 48: 92-99. 
21 Sussman C, Swanson G. Utility of the Sussman Wound Healing Tool in predicting wound healing outcomes in physical therapy. Adv Wound Care 1997; 10: 74-77.

22 Braden BJ. The Braden Scale for predicting pressure sore risk: reflections after 25 years. Adv Skin Wound Care 2012; 25: 61.

23 Woolrich RA, Kennedy P, Tasiemski T. A preliminary psychometric evaluation of the Hospital Anxiety and Depression Scale (HADS) in 963 people living with a spinal cord injury. Psychol Health Med 2006; 11: 80-90.

24 Zigmond AS, Snaith RP. The Hospital Anxiety and Depression Scale. Acta Psychiatr Scand 1983; 67: 361-370

25 Üstün TB, Kostanjsek N, Chatterii S, Rehm J. (eds) Measuring health and disability: manual for WHO Disability Assessment Schedule (WHODAS 2.0). WHO Press: Geneva, Switzerland, 2010.

26 Brooks R, De Charro F. EuroQol: the current state of play. Health Policy 1996; 37: 53-72.
27 Whitehurst DGT, Noonan VK, Dvorak MFS, Bryan S. A review of preference-based health-related quality of life questionnaires in spinal cord injury research. Spinal Cord 2012; 50: 646-654.

28 Ferreira-Valente MA, Pais-Ribeiro JL, Jensen MP. Validity of four pain intensity rating scales. Pain 2011; 152: 2399-2404.

29 Harris PA, Taylor R, Thielke R, Payne J, Gonzalez N, Conde JG. Research electronic data capture (REDCap)-a metadata-driven methodology and workflow process for providing translational research informatics support. J Biomed Inform 2009; 42 : 377-381.

30 Kirshblum SC, Burns SP, Biering-Sorensen F, Donovan W, Graves DE, Jha A et al. International standards for neurological classification of spinal cord injury (revised 2011). J Spinal Cord Med 2011; 34: 535-546.

31 Khor HM, Tan J, Saedon NI, Kamaruzzaman SB, Chin AV, Poi PJ et al. Determinants of mortality among older adults with pressure ulcers. Arch Gerontol Geriatr 2014; 59: 536-541. 